\title{
Benchmarking and optimization of a Next Generation Sequencing based method for transgene Sequence Variant Analysis in Biotherapeutic Cell Line Development
}

\author{
Joost Groot $^{1}$, Yizhou Zhou ${ }^{1}$, Eric Marshall ${ }^{1}$, Thomas Carlile ${ }^{1}$, Patrick Cullen ${ }^{1}$, Dongdong \\ Lin $^{1}$, Chongfeng Xu ${ }^{2}$, Justin Crisafulli ${ }^{1}$, Chao Sun ${ }^{1}$, Fergal Casey ${ }^{1}$, Baohong Zhang ${ }^{1}$, and \\ Christina Alves ${ }^{1}$ \\ ${ }^{1}$ Biogen Inc \\ ${ }^{2}$ Biogen
}

October 29, 2020

\begin{abstract}
In recent years Next-Generation Sequencing (NGS) based methods to detect mutations in biotherapeutic transgene products have become a key quality step deployed during the development of manufacturing cell line clones. Previously we reported on a higher throughput, rapid mutation detection method based on amplicon sequencing (targeting transgene RNA) and detailed its implementation to facilitate cell line clone selection. By gaining experience with our assay in a diverse set of cell line development programs, we improved the computational analysis as well as experimental protocols. Here we report on these improvements as well as on a comprehensive benchmarking of our assay. We evaluated assay performance by mixing amplicon samples of a verified mutated antibody clone with a non-mutated antibody clone to generate spike-in mutations from $60 \%$ down to $\sim 0.3 \%$ frequencies. We subsequently tested the effect of 16 different sample and NGS library preparation protocols on the assay's ability to quantify mutations and on the occurrence of false-positive background error mutations (artifacts). Our evaluation confirmed assay robustness, established a high confidence limit of detection of $\sim 0.6 \%$, and identified protocols that reduce error levels thereby significantly reducing a source of false positives that bottlenecked the identification of low-level true mutations.
\end{abstract}

\section{Hosted file}

Groot_Zhou_Transgene_Seq_Benchmarking_SUBMISSION_26oct2020.pdf available at https://authorea . com/users/371317/articles/489681-benchmarking-and-optimization-of-a-next-generationsequencing-based-method-for-transgene-sequence-variant-analysis-in-biotherapeutic-cellline-development 